\title{
IncRNA MEG8 is downregulated in osteoarthritis and regulates chondrocyte cell proliferation, apoptosis and inflammation
}

\author{
WEI XIE, LUOYONG JIANG, XIAOYANG HUANG, HONGXI SHANG, MINGHONG GAO, \\ WEI YOU, JIFENG TAN, HONG YAN and WEI SUN \\ Department of Orthopedics, The First Affiliated Hospital of Shenzhen University, \\ Shenzhen Second People's Hospital, Shenzhen, Guangdong 518000, P.R. China
}

Received June 22, 2020; Accepted April 16, 2021

DOI: $10.3892 /$ etm.2021.10587

\begin{abstract}
Long noncoding RNA (lncRNA) maternally expressed 8, small nucleolar RNA host gene (MEG8) has been widely reported for its pro-proliferative, anti-apoptotic and anti-inflammatory effects in diverse diseases. The aim of the present study was to investigate the effects and underlying mechanism of MEG8 on IL-1 $\beta$-stimulated human osteoarthritis (OA) chondrocytes. C28/I2 chondrocytes were cultured under the stimulation of IL-1 $\beta$ to establish a cellular model of OA. Functional assays involving Cell Counting Kit-8 and flow cytometry were performed to determine proliferation and apoptosis in the cells. The protein expression levels of caspase- 3 and inflammatory cytokines were detected using cell-based ELISA. The expression levels of PI3K/AKT pathway-related proteins were evaluated by western blotting. It was identified that MEG8 expression was increased in the cartilage of patients with OA and in IL-1 $\beta$-treated C28/I 2 cells. In C28/I2 cells, silencing of MEG8 expression noticeably triggered IL-1 $\beta$-induced proliferation suppression, cell death and an inflammatory response. However, transfection with MEG8 displayed adverse effects. Furthermore, MEG8 overexpression prevented IL-1 $\beta$-induced activation of the PI3K/AKT signaling pathway in C28/I2 cells. These data demonstrated that MEG8 exerted protective effects against IL-1 $\beta$-induced apoptosis and inflammation of OA chondrocytes by regulating the PI3K/AKT signaling pathway. Thus, the present study demonstrates that MEG8 might be a promising target for the treatment of OA.
\end{abstract}

Correspondence to: Dr Wei Sun, Department of Orthopedics, The First Affiliated Hospital of Shenzhen University, Shenzhen Second People's Hospital, 3002 Sungang West Road, Shenzhen, Guangdong 518000, P.R. China

E-mail: sunwei@webmailcn.com

Key words: apoptosis, inflammation, maternally expressed 8, small nucleolar RNA host gene, osteoarthritis, PI3K/AKT signaling pathway

\section{Introduction}

Osteoarthritis (OA) is a chronic joint disease involving articular cartilage erosion, osteophyte formation, subchondral sclerosis, and morphological changes in the synovium and articular capsules (1). OA represents a leading cause of physical disability, affecting the elderly population $(2,3)$. The known risk factors of OA include aging, acute trauma, obesity, chronic overload and genetics (4). Joint-preserving interventions including lifestyle modification, surgery and pharmaceutical drugs have been widely recommended (5). However, no effective therapy is available to actually modify disease progression. During the course of OA progression, regeneration of articular cartilage or prevention of further degeneration represent promising treatment strategies for preventing OA (6).

In recent years, a novel player, long noncoding RNA (lncRNA), a class of non-coding RNA products with transcript length $>200$ nucleotides, has been discovered to regulate biological functions (7). As a result of in-depth research into IncRNAs, it has been found that lncRNAs also play an important role in the pathogenesis of immune system diseases, respiratory system diseases, diabetes, heart disease and other chronic diseases (8). A number of recent studies provided strong evidence that aberrantly expressed lncRNAs are critical for chondrocyte dysfunction, including abnormal apoptosis and inflammatory response $(9,10)$. Maternally expressed 8 , small nucleolar RNA host gene (MEG8) is a small nucleolar RNA host gene, located at chromosome 14q32.3. Recently, the regulatory roles of MEG8 have been investigated in lung and pancreatic cancer, atherosclerosis trophoblast dysfunction and in liver fibrosis (11-14). Furthermore, a previous study referred to abnormal MEG8 expression in patients with OA (15). However, to the best of our knowledge, the involvement of MEG8 in OA has not been extensively investigated.

Previous studies have suggested that the PI3K/AKT signaling pathway may be involved in the regulation of chondrocyte apoptosis $(16,17)$. Previous studies found that human C28/I2 chondrocytes treated with IL-1 $\beta$ had significantly increased expression levels of phosphorylated (p)-PI3K and $\mathrm{p}-\mathrm{AKT}$ (18). PI3K/AKT is involved in the inflammatory response and is activated in OA progression (19). Accumulating studies have reported that inhibition of the PI3K/AKT signaling 
Table I. Baseline characteristics of patients with osteoarthritis.

\begin{tabular}{lc}
\hline Clinicopathological feature & Number of patients, $\mathrm{n}$ \\
\hline Sex & \\
Female & 10 \\
Male & 12 \\
Disease duration (years) & \\
$<5$ & 15 \\
$\geq 5$ & 7 \\
Kellgren-Lawrence stage & \\
III & 12 \\
IV & 10 \\
\hline
\end{tabular}

pathway alleviates the inflammatory response, reducing the levels of pro-inflammatory cytokines (20). Therefore, the inactivation of the PI3K/AKT signaling pathway may help delay OA progression.

Thus, the aim of the present study was to investigate the detailed effects of MEG8 silencing or overexpression on the proliferation, apoptosis and inflammatory response in IL-1 $\beta$-treated chondrocytes by regulating the PI3K/AKT signaling pathway.

\section{Materials and methods}

Tissue samples. Articular cartilage tissue samples were collected from patients with OA (age, 62 \pm 5 years) undergoing artificial knee arthroplasty, and from trauma patients without OA (age, $42 \pm 4$ years) who served as the healthy control ( $n=22$ per group). Samples were collected from The First Affiliated Hospital of Shenzhen University, Shenzhen Second People's Hospital (Shenzhen, China) from January 2018 to January 2019. The inclusion criteria were as follows: i) Patients who were diagnosed as osteoarthritic; ii) patients who were willing to participate; and iii) patients who fully understood the experimental protocol. The exclusion criteria were as follows: i) Patients with other disease complications; ii) patients who had been treated within the 3 months before admission; and iii) patients with a history of joint surgery. The clinical features of patients with OA including sex, disease duration and Kellgren-Lawrence stage (21) are presented in Table I. Informed consent was obtained from all patients and the present study was approved by the Ethics Committee of The First Affiliated Hospital of Shenzhen University, Shenzhen Second People's Hospital.

Cell culture, treatment and transfection. Human C28/I2 chondrocytes were purchased from The Cell Bank of Type Culture Collection of The Chinese Academy of Sciences and cultured in Dulbecco's modified Eagle's medium/Nutrient Mixture F-12 medium (Thermo Fisher Scientific, Inc.) containing 10\% fetal bovine serum and $100 \mathrm{U} / \mathrm{ml}$ penicillin-streptomycin solution (Gibco; Thermo Fisher Scientific, Inc.) in a humidified atmosphere with $5 \% \mathrm{CO}_{2}$ at $37^{\circ} \mathrm{C}$. For induction of the $\mathrm{OA}$ cellular model, C28/I2 cells were stimulated with $10 \mathrm{ng} / \mathrm{ml} \mathrm{IL-1 \beta}$ for $24 \mathrm{~h}$, as previously reported $(22,23)$. The MEG8 overexpression vector was generated by inserting its corresponding full-length sequence into a pcDNA3.1 vector (Thermo Fisher Scientific, Inc.) with a pcDNA3.1 empty vector serving as the negative control (NC). The small interfering (si)RNA against MEG8 and scrambled siRNA NC (si-NC) were generated by Chang Jing Bio-Tech, Ltd. The sequences were as follows: si-MEG8 sense, 5'-GGAAUAGACGAGAUUGGAUTT-3', and antisense, 5'-AUCCAAUCUCGUCUAUUCCTT-3'; si-NC, sense, 5'-UUCUCCGAACGUGUCACGUTT-3', and antisense, 5'-ACGUGACACGUUCGGAGAATT-3'. For cell transfection, the $\mathrm{C} 28 / \mathrm{I} 2$ cells were seeded into six-well plates and grown to cell density of $70 \%$, followed by transfection of $50 \mathrm{nM}$ oligonucleotides or $1 \mu \mathrm{g}$ vector using Lipofectamine ${ }^{\circledR}$ 2000 (Invitrogen; Thermo Fisher Scientific, Inc.).

Reverse transcription-quantitative PCR (RT-qPCR). Total RNA was extracted from cartilage tissue samples and C28/I2 cells using TRIzol ${ }^{\circledR}$ reagent (Invitrogen; Thermo Fisher Scientific, Inc.). Subsequently, $1 \mu \mathrm{g}$ RNA was reverse transcribed into cDNA using a First-strand cDNA Synthesis Kit (Tiangen Biotech Co., Ltd.) according to the manufacturer's instructions. To quantify the expression of MEG8, qPCR was performed using a SYBR ${ }^{\circledR}$ Premix Ex Taq ${ }^{\mathrm{TM}}$ kit (Takara Bio, Inc.). The thermocycling conditions were as follows: $95^{\circ} \mathrm{C}$ for $10 \mathrm{~min} ; 40$ cycles of $95^{\circ} \mathrm{C}$ for $15 \mathrm{sec} ; 60^{\circ} \mathrm{C}$ for $30 \mathrm{sec}$. The results are presented as fold-changes relative to $\mathrm{U} 6$ and were calculated using the $2^{-\Delta \Delta C q}$ method (24). The primers used were as follows: MEG8 forward, 5'-CAGTGTTGCCTGGGT CTGA-3' and reverse, 5'-ATCCCCTTGAAAGAGCAGGA-3'; GAPDH forward, 5'-CCACGAAACTACCTTCAACTC-3' and reverse, 5'-TCATACTCCTGCTGCTTGCTGATCC-3'.

Cell viability assay. Cell Counting Kit-8 (CCK-8; Beyotime Institute of Biotechnology) was used to detect cell viability according to the manufacturer's instructions. After IL-1 $\beta$ treatment or transfection, C28/I2 cells were inoculated onto 96 -well plates $\left(1 \times 10^{4}\right.$ cells/well) and cultured for $48 \mathrm{~h}$. Subsequently, $10 \mu \mathrm{l}$ CCK-8 solution was added, followed by incubation at $37^{\circ} \mathrm{C}$ for $1 \mathrm{~h}$. The absorbance of each well was analyzed at a wavelength of $450 \mathrm{~nm}$ using a Microplate Reader (BioTek Instruments, Inc.).

Flow cytometry assay. Cell apoptosis was analyzed by flow cytometry. After IL- $1 \beta$ treatment or transfection, C28/I2 cells were washed with PBS twice and resuspended in binding buffer, followed by double staining using an Annexin V-FITC/PI Apoptosis Detection Kit (Thermo Fisher Scientific, Inc.) in the dark for $15 \mathrm{~min}$. The percentages of early apoptotic and late apoptotic cells were analyzed by flow cytometry (BD Biosciences). The lower left quadrants represent viable cells, the lower right quadrants represent early apoptotic cells, the upper right quadrants represent late apoptotic cells and the upper left quadrants represent necrotic cells. The cells in the lower and upper right quadrants were summed to obtain the total number of apoptotic cells.

TUNEL staining. Apoptosis was detected by TUNEL staining, according to the instructions of the TUNEL kit (Beyotime Institute of Biotechnology). Briefly, slides were 
A

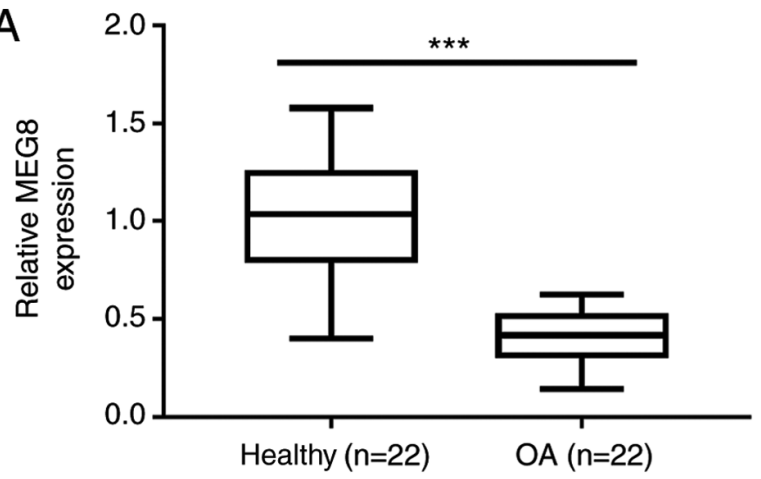

B

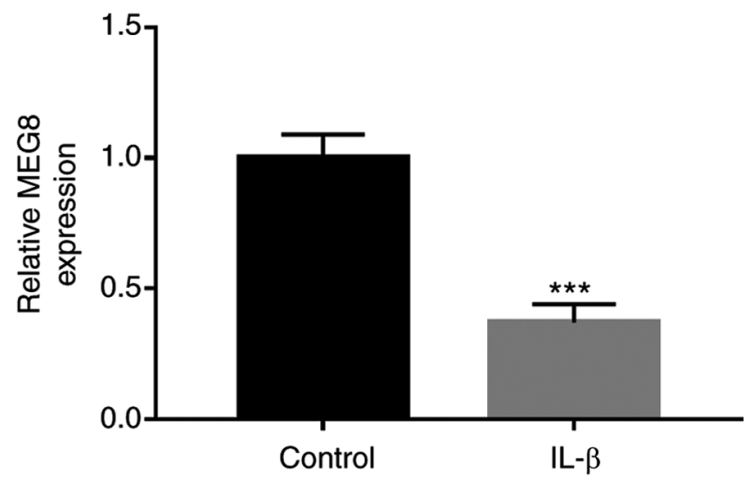

Figure 1. MEG8 expression is downregulated in OA cartilage and IL-1 $\beta$-induced chondrocytes. (A) The expression levels of MEG8 in OA and healthy tissue samples ( $\mathrm{n}=22$ per group) were evaluated by RT-qPCR. (B) MEG8 expression was detected by RT-qPCR in C28/I2 cells after stimulation with 10 ng/ml IL-1 $\beta$ for 24 h. ${ }^{* * *} \mathrm{P}<0.001$. MEG8, maternally expressed 8, small nucleolar RNA host gene; OA, osteoarthritis; RT-qPCR, reverse transcription-quantitative PCR.

permeabilized with $0.2 \%$ Triton-X, fixed with $4 \%$ paraformaldehyde and stained with the reaction mix. The color was developed with 3,3'-diaminobenzidine. Slides were mounted onto glass slides and cell death was determined by calculating the number of cells that showed positive TUNEL staining using a Laser Scanning Confocal Microscope (Leica Microsystems $\mathrm{GmbH}$ ).

Caspase-3 activity detection. Caspase-3 activity was determined using the caspase-3 activity kit (cat. no. BF3100; R\&D Systems, Inc.) according to the manufacturer's instructions. After IL-1 $\beta$ treatment or transfection, cells $\left(5 \times 10^{6}\right)$ were resuspended with cold lysis buffer. Cell lysates were then collected and incubated in reaction buffer containing substrates for $1 \mathrm{~h}$ in the dark at $37^{\circ} \mathrm{C}$. The absorbance was detected at a wavelength of $405 \mathrm{~nm}$.

Enzyme-linked immunosorbent assay (ELISA). The inflammatory response was determined by the secretion of inflammatory cytokines using a commercial ELISA kit (MilliporeSigma) according to the manufacturer's instructions. After IL-1 $\beta$ treatment or transfection, the cell medium was collected and the concentrations of TNF- $\alpha$ and IL-6 in the supernatant were analyzed. The absorbance was analyzed at a wavelength of $450 \mathrm{~nm}$.

Western blotting. Following IL-1 $\beta$ treatment or transfection, the total proteins of C28/I2 cells were extracted using RIPA lysis (Thermo Fisher Scientific, Inc.) and the protein content was evaluated using a BCA Protein Assay Kit (Beyotime Institute of Biotechnology). Proteins (30 $\mu \mathrm{g}$ per lane) were separated by electrophoresis on $15 \%$ SDS-PAGE gels and transferred to polyvinylidenedifluoride membranes. Afterwards, membranes were blocked at room temperature for $1 \mathrm{~h}$ with $5 \%$ skimmed milk powder, and then incubated with primary antibodies against AKT (cat. no. \#4685), p-AKT (cat. no. \#4060), PI3K (cat. no. \#4249) and p-PI3K (cat. no. \#17366) (1:1,000 dilution; all from Cell Signaling Technology, Inc.) overnight at $4^{\circ} \mathrm{C}$. After being washed with phosphate-buffered saline three times for $5 \mathrm{~min}$, they were incubated with goat anti-rabbit IgG H\&L (HRP) secondary antibodies (cat. no. \#7074; 1:2,000 dilution; Cell Signaling Technology, Inc.) for $1 \mathrm{~h}$ at room temperature according to the manufacturer's instructions. Protein bands were visualized by enhanced chemiluminescence (Beyotime Institute of Biotechnology). Densitometric measurements were performed using ImageJ version 1.48 Software (National Institutes of Health).

Statistical analysis. All experiments were performed three times and data are expressed as means \pm standard deviation. Statistical analysis was conducted using GraphPad Prism 7 software (GraphPad, Inc.). Unpaired or paired (for tissue samples) Student's t-tests were used for comparisons between two groups and one-way analysis of variance with Tukey's post hoc test was used to compare three or more groups. $\mathrm{P}<0.05$ was considered to indicate a statistically significant difference.

\section{Results}

MEG8 is downregulated in OA cartilage and IL-1 $\beta$-induced chondrocytes. To investigate whether MEG8 is involved in OA, MEG8 expression levels in OA and healthy cartilage tissue samples were investigated. As shown in Fig. 1A, the expression level of MEG8 in OA tissue samples was markedly lower than that in the healthy controls. To establish the cellular model of OA, C28/I2 chondrocyte cells were stimulated with IL-1 $\beta$. The results indicate that IL-1 $\beta$ treatment significantly induced the downregulated expression of MEG8 (Fig. 1B).

MEG8 mitigates IL-1 $\beta$-induced chondrocyte injury. To investigate the biological role of MEG8 in OA, MEG8 expression was suppressed by transfecting C28/I2 cells with si-MEG8 (Fig. 2A). As demonstrated by CCK-8 and flow cytometry assays, the IL-1 $\beta$-induced decrease in cell viability (Fig. 2B) and increase in apoptosis (Fig. 2C and D) in C28/I2 cells were all promoted by MEG8 knockdown. Apoptosis is indicated by positive TUNEL staining in apoptotic cell death. Fig. 2E demonstrates the fluorescent signal of TUNEL staining of C28/I2 cells in the IL-1 $\beta$-induced OA cell model. In the untreated group, there was a small number of dead cells, whereas the rate of cell death was significantly increased following MEG8 silencing. In addition, the ELISA results indicated that MEG8 suppression upregulated caspase- 3 activity in IL-1 $\beta$-treated cells (Fig. 2F). The effects of MEG8 downregulation on the production of pro-inflammatory factors were also evaluated. As shown in Fig. $2 \mathrm{G}$ and H, the expression levels of TNF- $\alpha$ and 

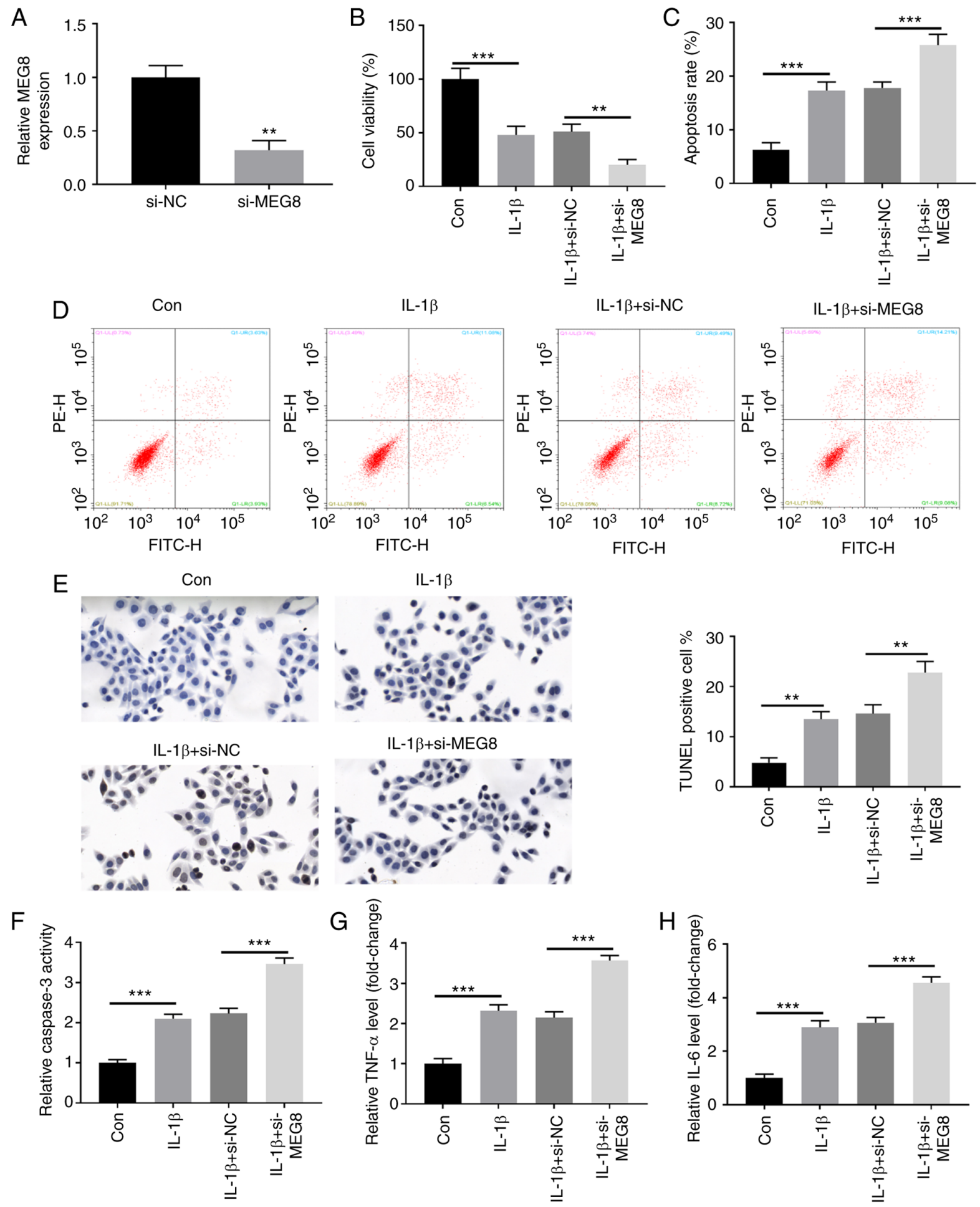

Figure 2. Effect of MEG8 knockdown on IL-1 $\beta$-induced chondrocyte injury. C28/I2 cells were transfected with si-MEG8. (A) MEG8 expression was assessed by reverse transcription-quantitative PCR. Following treatment with IL-1 $\beta$, cell viability and apoptosis were analyzed by (B) Cell Counting Kit- 8 assay and $(C$ and D) flow cytometry. (E) TUNEL staining was performed to detect the number of dead cells in IL-1 $\beta$-treated C28/I2 cells that underwent transfection with si-NC or si-MEG8 (magnification, x100). (F) Caspase-3 activity was examined by ELISA assay following treatment with IL-1 $\beta$. The concentrations of (G) TNF- $\alpha$ and (H) IL-6 in the culture supernatant were determined by ELISA assay, following treatment with IL-1 $1 \beta .{ }^{* * *} \mathrm{P}<0.01$ and ${ }^{* * *} \mathrm{P}<0.001$. MEG8, maternally expressed 8, small nucleolar RNA host gene; si, small interfering; NC, negative control; Con, control.

IL-6 were significantly elevated in IL-1 $\beta$-stimulated C28/I2 cells and these results were further enhanced by depletion of MEG8 expression.

Fig. 3A indicates that MEG8 was successfully overexpressed in C28/I2 cells by transfection with MEG8 expression vectors. Furthermore, overexpression of MEG8 alleviated the
IL-1 $\beta$-induced proliferation inhibition and apoptosis induction (Fig. 3B-D). In the TUNEL assay, as expected, MEG8 overexpression inhibited apoptosis induced by IL-1 $\beta$ (Fig. 3E). Furthermore, the IL-1 $\beta$ treatment-stimulated increase in caspase- 3 activity and production of TNF- $\alpha$ and IL- 6 were reversed by restoration of MEG8 expression (Fig. 3F-H). Thus, 
A

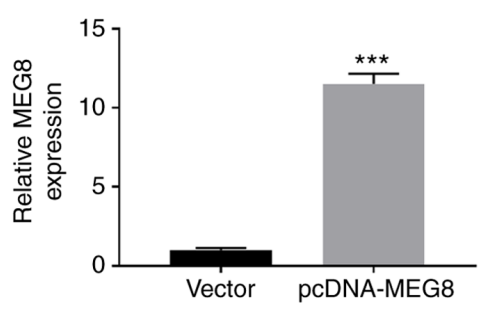

D

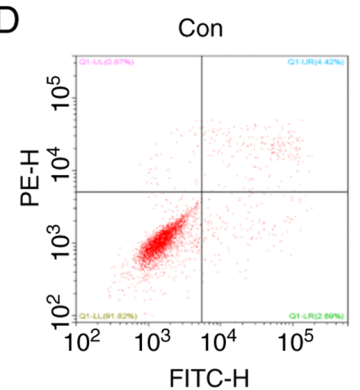

B

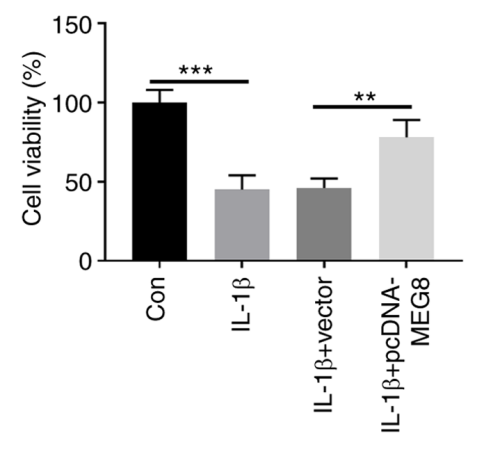

IL-1 $\beta$

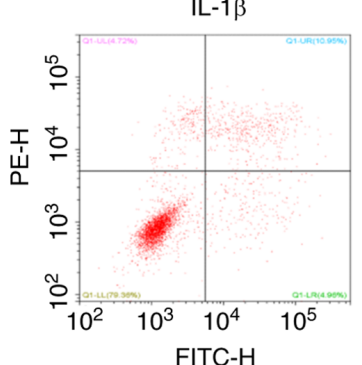

C

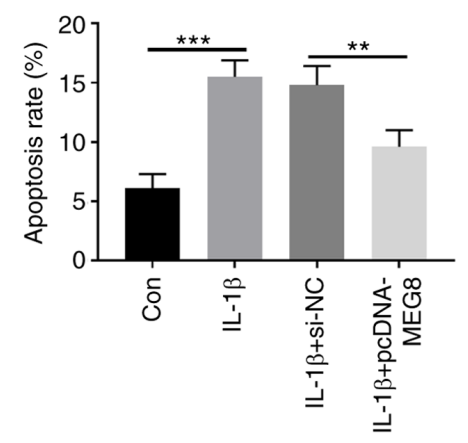

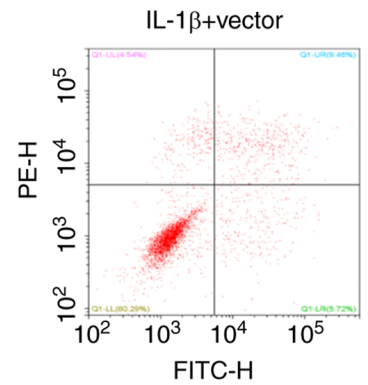

IL-1 $\beta+p c D N A-M E G 8$

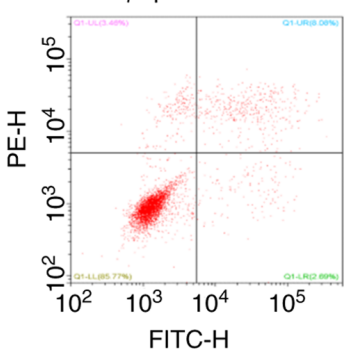

E

Con

$\mathrm{IL}-1 \beta$
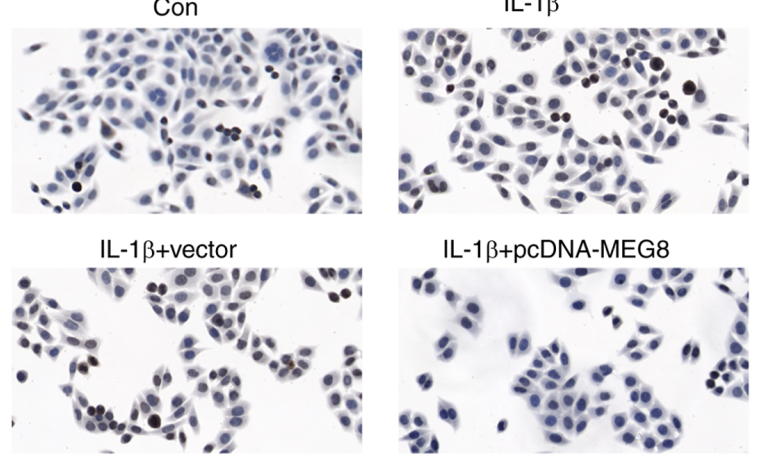

IL-1 $1 \beta+$ PCDNA-MEG8
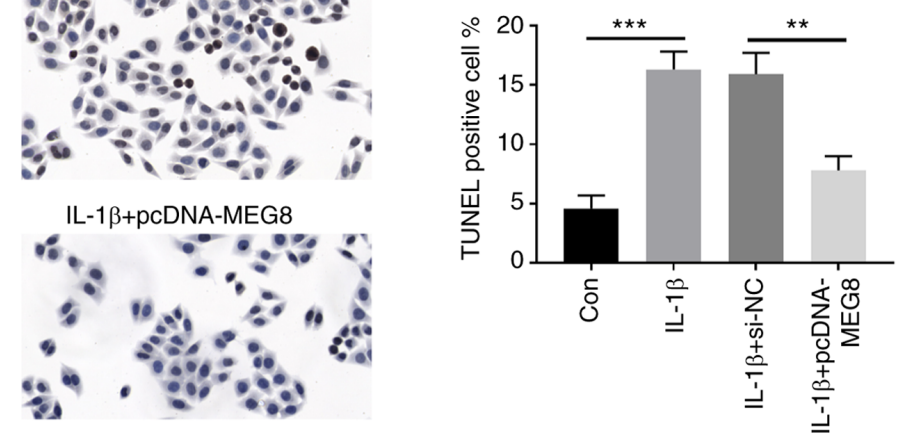

$\mathrm{F}$
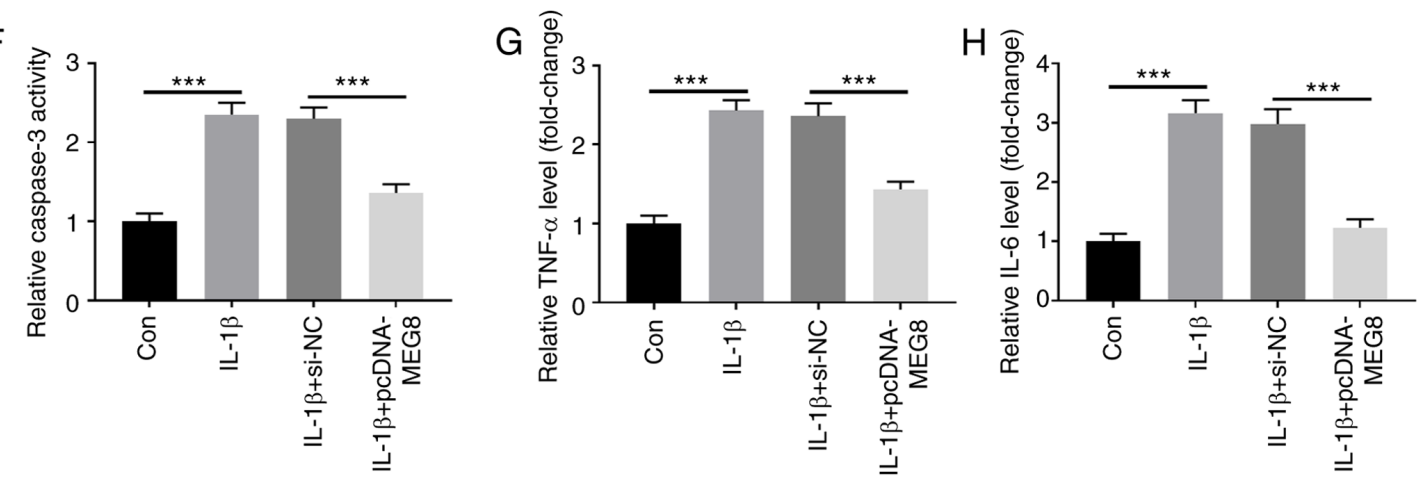

Figure 3. Effect of MEG8 overexpression on IL-1 $\beta$-induced chondrocyte injury. C28/I2 cells were transfected with MEG8 expressing vector. (A) MEG8 expression was assessed by reverse transcription-quantitative PCR. Cell viability and apoptosis were evaluated using (B) CCK-8 and (C and D) flow cytometry, following treatment with IL-1 $\beta$. (E) TUNEL staining was performed to detect the number of dead cells in IL-1 $\beta$-treated C28/I2 cells that underwent transfection with pcDNA3.1 empty vector or MEG8 expressing vector (magnification, x100). (F) Caspase-3 activity was examined by ELISA assay, following treatment with IL-1 $\beta$. The concentrations of $(\mathrm{G})$ TNF- $\alpha$ and $(\mathrm{H})$ IL- 6 in the culture supernatant were determined by ELISA assay following treatment with IL-1ß. ${ }^{* *} \mathrm{P}<0.01$ and ${ }^{* * *} \mathrm{P}<0.001$. MEG8, maternally expressed 8, small nucleolar RNA host gene; si, small interfering; Con, control.

these data indicated that MEG8 could relieve IL-1 $\beta$-induced cell apoptosis and inflammatory injury in C28/I2 cells.

MEG8 inhibits IL-1 $\beta$-induced activation of the PI3K/AKT signaling pathway in human chondrocytes. To further investigate the underlying mechanism of MEG8 in OA, whether
MEG8 affected PI3K/AKT signaling pathway-related protein expression was analyzed. The expression levels of p-PI3K and p-AKT were markedly increased in C28/I2 cells by treatment with IL- $1 \beta$, indicating that IL- $1 \beta$ could activate the PI3K/AKT signaling pathway in C28/I2 cells. However, the upregulation of PI3K and AKT phosphorylation induced 
A

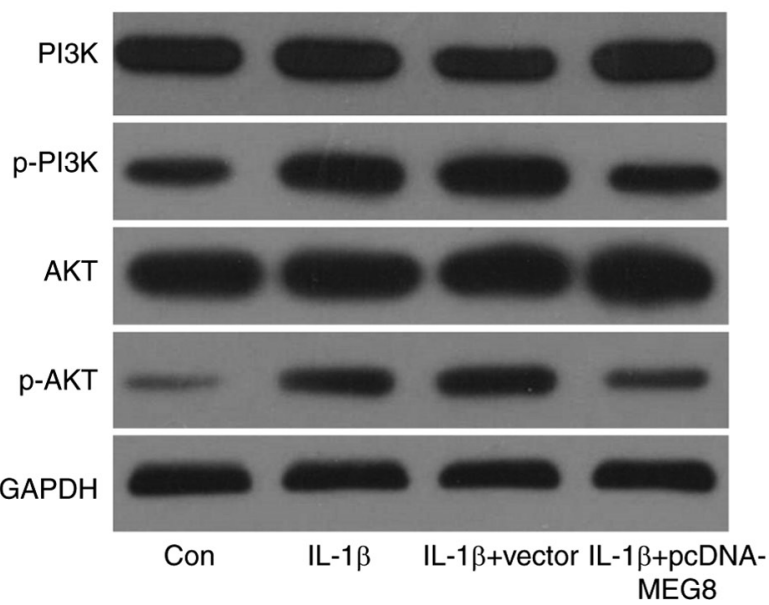

B

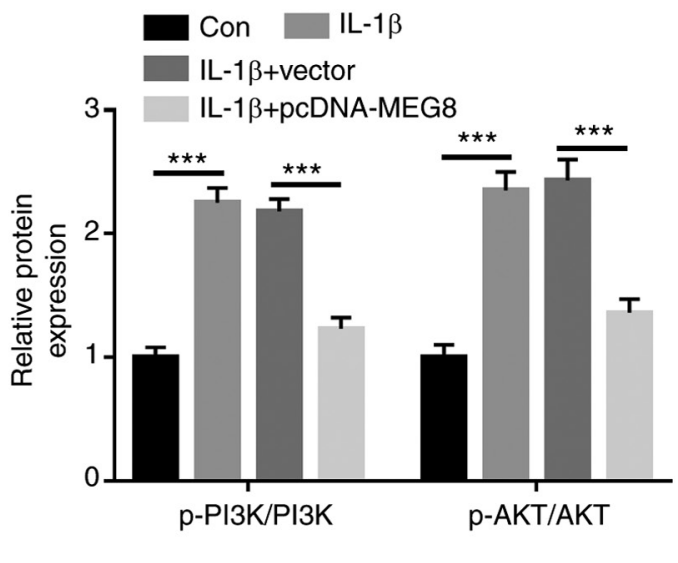

Figure 4. MEG8 inhibits IL-1 $\beta$-induced activation of the PI3K/AKT signaling pathway in human chondrocytes. (A and B) Western blot analysis of AKT, p-AKT, PI3K and p-PI3K protein expression levels in C28/I2 cells transfected with pcDNA3.1-MEG8 or pcDNA3.1 empty vector following treatment with IL-1 $\beta$. ${ }^{* * *} \mathrm{P}<0.001$. MEG8, maternally expressed 8, small nucleolar RNA host gene; p, phosphorylated; Con, control.

by IL-1 $\beta$ was significantly inhibited by ectopic expression of MEG8 in C28/I2 cells, implying that MEG8 activated the $\mathrm{PI} 3 \mathrm{~K} / \mathrm{AKT}$ signaling pathway in IL-1 $\beta$-treated chondrocytes (Fig. 4A and B).

\section{Discussion}

In the present study, the role and regulatory mechanism of MEG8 in the pathogenesis of OA was investigated. MEG8 expression was shown to be significantly decreased in OA cartilage and IL-1 $\beta$-treated chondrocytes. Moreover, functional exploration demonstrated that MEG8 induced cell proliferation while inhibiting apoptosis and inflammatory injury in IL-1 $\beta$-treated chondrocytes. The present study also indicated that MEG8 overexpression attenuated IL-1 $\beta$-induced cell injury via mediation of the PI3K/AKT signaling pathway. Thus, it was indicated that MEG8 might be an essential candidate for reversing OA progression.

In recent years, an increasing number of studies have shown that lncRNAs play pivotal roles in regulating the biological functions of chondrocytes in OA. For example, Chen et al (25) indicated that MEG3 promoted proliferation and alleviated apoptosis and extracellular matrix (ECM) degradation in IL-1 $\beta$-induced chondrocytes by acting as a sponge of microRNA (miR)-93. Wang et al (26) showed that FOXD2 adjacent opposite strand RNA 1 sponged miR-27a-3p to accelerate cell proliferation, inflammation and ECM degradation in IL-1 $\beta$ and/or TNF- $\alpha$-treated chondrocytes by targeting Toll-like receptor 4 . Li et al (27) reported that $\mathrm{X}$-inactive specific transcript (XIST) was significantly upregulated in OA, and knockdown of XIST relieved IL-1 $\beta$-inhibition of chondrocyte proliferation and promoted IL- $1 \beta$-induced cell apoptosis by regulating miR-211-mediated C-X-C motif chemokine receptor 4 and MAPK signaling pathways. Tian et al (28) demonstrated that small nucleolar RNA host gene 7 was involved in IL-1 $\beta$-induced chondrocyte apoptosis and autophagy by regulating the miR-34a-5p/synoviolin 1 axis. MEG8 has been shown to be implicated in a diverse range of biological processes, including embryonic development, carcinogenesis and osteoblast differentiation $(11,29,30)$.
Until recently, little was known about the potential role of MEG8 in OA. However, Fu et al (15) reported that the expression level of MEG8 was low in OA cartilage when compared with that in healthy samples.

In the present study, the level of MEG8 expression was observed to be markedly downregulated in OA cartilage and IL-1 $\beta$-treated C28/I 2 chondrocytes when compared to healthy tissue samples or untreated cells. Consistent with previous studies $(31,32)$, the present study found that cell viability was inhibited, while apoptosis and pro-inflammatory cytokine expression were enhanced in IL-1 $\beta$-stimulated C28/I 2 cells. The inhibition of MEG8 by siRNA could facilitate chondrocyte apoptosis caused by IL-1 $\beta$ and the production of pro-inflammatory cytokines, while ameliorating proliferation. However, the overexpression of MEG8 could partly reverse the acceleration of cell apoptosis and the inflammatory response, as well as the anti-proliferative effect caused by IL- $1 \beta$ in chondrocytes. Consequently, the abovementioned results indicated that MEG8 served as a suppressor of OA progression by accelerating proliferation and suppressing apoptosis and inflammatory responses in chondrocytes. However, the exact molecular mechanism by which MEG8 is implicated in OA remains unclear.

Compelling evidence has delineated the involvement of the PI3K/AKT signaling pathway in diverse cell processes, such as survival, migration, apoptosis, autophagy and differentiation (33-35). Several lines of evidence indicate that inhibition of the PI3K/AKT signaling pathway reduced the apoptosis and inflammatory response of articular chondrocytes $(36,37)$. Hence, to investigate the underlying mechanism of MEG8 in OA chondrocytes, the present study focused on the PI3K/AKT signaling pathway. MEG8 overexpression reversed IL- $1 \beta$-induced activation of the PI3K/AKT signaling pathway. These findings suggested that PI3K/AKT signaling was a critical target for OA and that MEG8 may harness its potential therapeutic effect by inhibiting this signaling pathway.

Thus, the present results showed that downregulation of MEG8 induced by IL-1 $\beta$ aggravated OA progression via activation of the PI3K/AKT signaling pathway. Due to the small 
sample size and the lack of an in vivo assay, further investigation of the MEG8/PI3K/AKT axis in OA is essential.

\section{Acknowledgements}

Not applicable.

\section{Funding}

No funding was received.

\section{Availability of data and materials}

The datasets used and/or analyzed during the current study are available from the corresponding author on reasonable request.

\section{Authors' contributions}

WS, JT and WX designed the study; MG, HY, WX, LJ, XH, HS and WY performed the experiments, analyzed the data and prepared the manuscript. WX, MG, JT and HY reviewed the manuscript. All authors confirmed the authenticity of all the raw data. All authors have read and approved the final manuscript.

\section{Ethics approval and consent to participate}

The present study was approved by the ethic committee of The First Affiliated Hospital of Shenzhen University, Shenzhen Second People's Hospital. Written informed consent was obtained from all participants.

\section{Patient consent for publication}

Not applicable.

\section{Competing interests}

The authors declare that they have no competing interests.

\section{References}

1. Pereira D, Ramos E and Branco J: Osteoarthritis. Acta Med Port 28: 99-106, 2015.

2. Neogi T and Zhang Y: Epidemiology of osteoarthritis. Rheum Dis Clin North Am 39: 1-19, 2013.

3. Vina ER and Kwoh CK: Epidemiology of osteoarthritis: Literature update. Curr Opin Rheumatol 30: 160-167, 2018.

4. He Y, Li Z, Alexander PG, Ocasio-Nieves BD, Yocum L, Lin H and Tuan RS: Pathogenesis of osteoarthritis: Risk factors, regulatory pathways in chondrocytes, and experimental models. Biology (Basel) 9: 194, 2020.

5. Glyn-Jones S, Palmer AJ, Agricola R, Price AJ, Vincent TL, Weinans H and Carr AJ: Osteoarthritis. Lancet 386: 376-387, 2015.

6. Liao J and Lin Y: Stem cells and cartilage tissue engineering. Curr Stem Cell Res Ther 13: 489, 2018.

7. Sanchez Calle A, Kawamura Y, Yamamoto Y, Takeshita F and Ochiya T: Emerging roles of long non-coding RNA in cancer. Cancer Sci 109: 2093-2100, 2018.

8. Mishra S, Verma SS, Rai V, Awasthee N, Chava S, Hui KM, Kumar AP, Challagundla KB, Sethi G and Gupta SC: Long non-coding RNAs are emerging targets of phytochemicals for cancer and other chronic diseases. Cel Mol Life Sci 76: 1947-1966, 2019
9. Liang Z and Ren C: Emodin attenuates apoptosis and inflammation induced by LPS through up-regulating lncRNA TUG1 in murine chondrogenic ATDC5 cells. Biomed Pharmacother 103: 897-902, 2018

10. Pan L, Liu D, Zhao L, Wang L, Xin M and Li X: Long noncoding RNA MALAT1 alleviates lipopolysaccharide-induced inflammatory injury by upregulating microRNA-19b in murine chondrogenic ATDC5 cells. J Cell Biochem 119: 10165-10175, 2018.

11. Terashima M, Ishimura A, Wanna-Udom S and Suzuki T: MEG8 long noncoding RNA contributes to epigenetic progression of the epithelial-mesenchymal transition of lung and pancreatic cancer cells. J Biol Chem 293: 18016-18030, 2018.

12. Zhang B, Dong Y and Zhao Z: IncRNA MEG8 regulates vascular smooth muscle cell proliferation, migration and apoptosis by targeting PPAR $\alpha$. Biochem Biophys Res Commun 510: 171-176, 2019.

13. Sheng F, Sun N, Ji Y, Ma Y, Ding H, Zhang Q, Yang F and Li W: Aberrant expression of imprinted lncRNA MEG8 causes trophoblast dysfunction and abortion. J Cell Biochem 120: 17378-17390, 2019.

14. Chen T, Lin H, Chen X, Li G, Zhao Y, Zheng L, Shi Z, Zhang K, Hong W and Han T: lncRNA Meg8 suppresses activation of hepatic stellate cells and epithelial-mesenchymal transition of hepatocytes via the Notch pathway. Biochem Biophys Res Commun 521: 921-927, 2020.

15. Fu M, Huang G, Zhang Z, Liu J, Zhang Z, Huang Z, Yu B and Meng F: Expression profile of long noncoding RNAs in cartilage from knee osteoarthritis patients. Osteoarthritis Cartilage 23: 423-432, 2015.

16. Li H, Xie S, Li H, Zhang R and Zhang H: lncRNA MALAT1 mediates proliferation of LPS treated-articular chondrocytes by targeting the miR-146a-PI3K/Akt/mTOR axis. Life Sciences 254: 116801,2020

17. Fan Z, Liu Y, Shi Z, Deng K, Zhang H, Li Q, Cao S, Li S and Zhang H: MiR-155 promotes interleukin-1 $\beta$-induced chondrocyte apoptosis and catabolic activity by targeting PIK3R1-mediated PI3K/Akt pathway. J Cell Mol Med 24: 8441-8451, 2020.

18. Wang Y, Shen S, Li Z, Li W and Weng X: MIR-140-5p affects chondrocyte proliferation, apoptosis, and inflammation by targeting HMGB1 in osteoarthritis. Inflamm Res 69: 63-73, 2020.

19. Xie L, Xie H, Chen C, Tao Z, Zhang $C$ and Cai L: Inhibiting the $\mathrm{PI} 3 \mathrm{~K} / \mathrm{AKT} / \mathrm{NF}-\kappa \mathrm{B}$ signal pathway with nobiletin for attenuating the development of osteoarthritis: In vitro and in vivo studies. Food Funct 10: 2161-2175, 2019.

20. Wu M, Hu R, Wang J, An Y, Lu L, Long C and Yan L: Salidroside Suppresses IL-1 $\beta$-induced apoptosis in chondrocytes via phosphatidylinositol 3-kinases (PI3K)/Akt signaling inhibition. Med Sci Monit 25: 5833-5840, 2019.

21. Luyten FP, Bierma-Zeinstra S, Dell'Accio F, Kraus VB, Nakata K, Sekiya I, Arden NK and Lohmander LS: Toward classification criteria for early osteoarthritis of the knee. Semin Arthritis Rheum 47: 457-463, 2018.

22. Lei J, Fu Y, Zhuang Y, Zhang K and Lu D: lncRNA SNHG1 alleviates IL-1 $\beta$-induced osteoarthritis by inhibiting miR-16-5pmediated p38 MAPK and NF- $\mathrm{BB}$ signaling pathways. Biosci Rep 39: BSR20191523, 2019.

23. Lu X, Yu Y, Yin F, Yang C, Li B, Lin J and Yu H: Knockdown of PVT1 inhibits IL-1 $\beta$-induced injury in chondrocytes by regulating miR-27b-3p/TRAF3 axis. Int Immunopharmacol 79: $106052,2020$.

24. Livak KJ and Schmittgen TD: Analysis of relative gene expression data using real-time quantitative PCR and the 2(-Delta Delta C(T)) method. Methods 25: 402-408, 2001.

25. Chen K, Zhu H, Zheng MQ and Dong QR: lncRNA MEG3 inhibits the degradation of the extracellular matrix of chondrocytes in osteoarthritis via targeting miR-93/TGFBR2 axis. Cartilage: Jun 28, 2019 (Epub ahead of print).

26. Wang Y, Cao L, Wang Q, Huang J and Xu S: lncRNA FOXD2-AS1 induces chondrocyte proliferation through sponging miR-27a-3p in osteoarthritis. Artif Cells Nanomed Biotechnol 47: 1241-1247, 2019.

27. Li L, Lv G, Wang B and Kuang L: The role of lncRNA XIST/miR-211 axis in modulating the proliferation and apoptosis of osteoarthritis chondrocytes through CXCR4 and MAPK signaling. Biochem Biophys Res Commun 503: 2555-2562, 2018.

28. Tian F, Wang J, Zhang Z and Yang J: lncRNA SNHG7/ miR-34a-5p/SY VN1 axis plays a vital role in proliferation, apoptosis and autophagy in osteoarthritis. Biol Res 53: 9, 2020. 
29. Gu T, He H, Han Z, Zeng T, Huang Z, Liu Q, Gu N, Chen Y, Sugimoto K, Jiang $\mathrm{H}$ and Wu Q: Expression of macro non-coding RNAs Meg8 and Irm in mouse embryonic development. Acta Histochem 114: 392-399, 2012.

30. Zheng Y, Li X, Huang Y, Jia L and Li W: Time series clustering of mRNA and IncRNA expression during osteogenic differentiation of periodontal ligament stem cells. PeerJ 6: e5214, 2018.

31. ZhouX,JiangL,FanG, Yang H,WuL,Huang Y,Xu NandLiJ:Role of the ciRS-7/miR-7 axis in the regulation of proliferation, apoptosis and inflammation of chondrocytes induced by IL-1 $\beta$. Int Immunopharmacol 71: 233-240, 2019.

32. Liu M, Zhong S, Kong R, Shao H, Wang C, Piao H, Lv W, Chu X and Zhao Y: Paeonol alleviates interleukin-1 $\beta$-induced inflammatory responses in chondrocytes during osteoarthritis. Biomed Pharmacother 95: 914-921, 2017.

33. Chai C, Song LJ, Han SY, Li XQ and Li M: MicroRNA-21 promotes glioma cell proliferation and inhibits senescence and apoptosis by targeting SPRY1 via the PTEN/PI3K/AKT signaling pathway. CNS Neurosci Ther 24: 369-380, 2018.

34. Zhang Q, Lai S, Hou X, Cao W, Zhang Y and Zhang Z: Protective effects of PI3K/Akt signal pathway induced cell autophagy in rat knee joint cartilage injury. Am J Transl Res 10 762-770, 2018
35. Wu R, Ruan J, Sun Y, Liu M, Sha Z, Fan C and Wu Q: Long non-coding RNA HIF1A-AS2 facilitates adipose-derived stem cells (ASCs) osteogenic differentiation through miR-665/IL6 axis via PI3K/Akt signaling pathway. Stem Cell Res Ther 9: 348, 2018.

36. Xue JF, Shi ZM,Zou J and Li XL: Inhibition of PI3K/AKT/mTOR signaling pathway promotes autophagy of articular chondrocytes and attenuates inflammatory response in rats with osteoarthritis. Biomed Pharmacother 89: 1252-1261, 2017.

37. Sun J, Song X, Su L and Cao S: Long non-coding RNA LncHIFCAR promotes osteoarthritis development via positively regulating HIF- $1 \alpha$ and activating the PI3K/AKT/mTOR pathway. Int J Clin Exp Pathol 11: 3000-3009, 2018. Attribution-NonCommercial-NoDerivatives 4.0 International (CC BY-NC-ND 4.0) License. 